\title{
Spin dynamics in the single-ion magnet $\left[\operatorname{Er}\left(\mathrm{W}_{5} \mathrm{O}_{18}\right)_{2}\right]^{9-}$
}

\author{
M. Mariani, ${ }^{1}$ F. Borsa, ${ }^{1,2}$ M. J. Graf, ${ }^{3}$ S. Sanna,,${ }^{1,4}$ M. Filibian, ${ }^{1}$ T. Orlando,,${ }^{1,5}$ K. P. V. Sabareesh, ${ }^{1,6}$ \\ S. Cardona-Serra, ${ }^{7}$ E. Coronado, ${ }^{7}$ and A. Lascialfari ${ }^{8,9,1}$ \\ ${ }^{1}$ Department of Physics, Università degli Studi di Pavia and INSTM, I-27100 Pavia, Italy \\ ${ }^{2}$ Department of Physics and Astronomy and Ames Laboratory, Iowa State University, Ames, Iowa 50011, USA \\ ${ }^{3}$ Department of Physics, Boston College, Chestnut Hill, Massachusetts 02467, USA \\ ${ }^{4}$ Department of Physics and Astronomy, Università di Bologna, I-40127 Bologna, Italy \\ ${ }^{5}$ Max Planck Institute for Biophysical Chemistry, I-37077 Gottingen, Germany \\ ${ }^{6}$ University of Information Science and Technology "St. Paul the Apostle," 6000 Ohrid, R. Macedonia \\ ${ }^{7}$ Instituto de Ciencia Molecular, Universitat de Valencia, 46980 Paterna, Spain \\ ${ }^{8}$ Department of Physics, Università degli Studi di Milano and INSTM, I-20133 Milano, Italy \\ ${ }^{9}$ Centre S3, clo Istituto Nanoscienze del CNR (CNR-NANO), I-41125 Modena, Italy
}

(Received 8 December 2017; revised manuscript received 15 March 2018; published 19 April 2018)

\begin{abstract}
In this work we present a detailed NMR and $\mu^{+} \mathrm{SR}$ investigation of the spin dynamics in the new hydrated sodium salt containing the single-ion magnet $\left[\mathrm{Er}\left(\mathrm{W}_{5} \mathrm{O}_{18}\right)_{2}\right]^{9-}$. The ${ }^{1} \mathrm{H}$ NMR absorption spectra at various applied magnetic fields present a line broadening on decreasing temperature which indicates a progressive spin freezing of the single-molecule magnetic moments. The onset of quasistatic local magnetic fields, due to spin freezing, is observed also in the muon relaxation curves at low temperature. Both techniques yield a local field distribution of the order of $0.1-0.2 \mathrm{~T}$, which appears to be of dipolar origin. On decreasing the temperature, a gradual loss of the ${ }^{1} \mathrm{H}$ NMR signal intensity is observed, a phenomenon known as wipe-out effect. The effect is analyzed quantitatively on the basis of a simple model which relies on the enhancement of the NMR spin-spin, $T_{2}^{-1}$, relaxation rate due to the slowing down of the magnetic fluctuations. Measurements of spin-lattice relaxation rate $T_{1}^{-1}$ for ${ }^{1} \mathrm{HNMR}$ and of the muon longitudinal relaxation rate $\lambda$ show an increase as the temperature is lowered. However, while for the NMR case the signal is lost before reaching the very slow fluctuation region, the muon spin-lattice relaxation $\lambda$ can be followed until very low temperatures and the characteristic maximum, reached when the electronic spin fluctuation frequency becomes of the order of the muon Larmor frequency, can be observed. At high temperatures, the data can be well reproduced with a simple model based on a single correlation time $\tau=\tau_{0} \exp (\Delta / \mathrm{T})$ for the magnetic fluctuations. However, to fit the relaxation data for both NMR and $\mu^{+} \mathrm{SR}$ over the whole temperature and magnetic field range, one has to use a more detailed model that takes into account spin-phonon transitions among the $\mathrm{Er}^{3+}$ magnetic sublevels. A good agreement for both proton NMR and $\mu^{+} \mathrm{SR}$ relaxation is obtained, which confirms the validity of the energy level scheme previously calculated from an effective crystal field Hamiltonian.
\end{abstract}

DOI: 10.1103/PhysRevB.97.144414

\section{INTRODUCTION}

In the last decades, a large effort has been devoted to the chemical design of low-dimensional molecular magnets with tailored magnetic properties suitable for the study of the nanoscale molecular magnetism and for different possible applications like magnetic storage, quantum computation, spintronics and so on [1,2]. One dimensional (1D) magnetic chains and zero-dimensional systems, i.e., magnetic molecular rings and clusters, have been thoroughly investigated to test theoretical models on real systems like, e.g., Glauber dynamics [3] in ferrimagnetic anisotropic magnetic chains [4-9] and Villain's conjecture [10-12] in fully frustrated quasi-onedimensional molecular magnets [13-17]. Furthermore, the study of molecular clusters has led to the discovery of new interesting effects such as quantum tunneling of the magnetization [18-22], the magnetic Berry phase [23], and the resonant phonon trapping mechanism for the magnetization relaxation [24].
Anyhow, the research on molecular magnetism has been dominated by wide-spread investigations of the so-called molecular nanomagnets or single-molecule-magnets (SMMs). From a chemical point of view, they are polynuclear metal complexes, which can act like individual, isolated magnets, since in the solid state these magnetic molecules are shielded from each other by a shell of bulky organic ligands leading to strong intramolecular exchange interactions among the magnetic centers and negligible inter-molecular magnetic interactions among molecules. With access to different chemical approaches, it is possible to synthesize a variety of complexes having various numbers and types of magnetic atoms in each molecule. The reduced number of magnetic atoms involved makes often possible to determine, through analytical or numerical methods, the structure of the discrete energy levels and consequently the physical properties of most molecular clusters.

The high-spin ground state and the uniaxial magnetic anisotropy [25] of SMMs lead to an extreme slowing down 


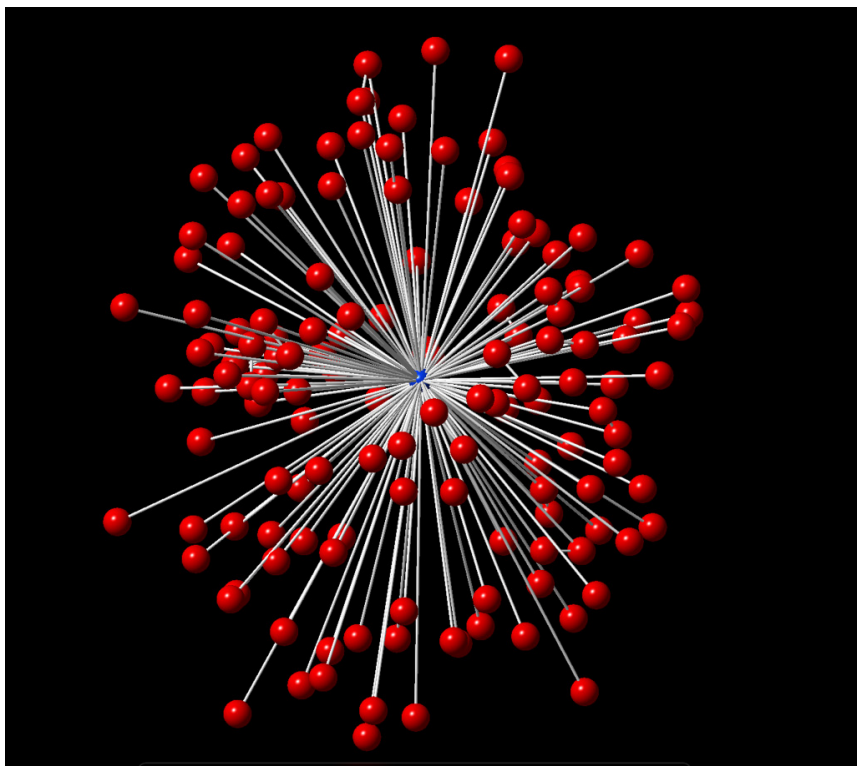

FIG. 1. Pictorial representation of the distribution of the water molecules (only the oxygens are shown) around the central $\mathrm{Er}^{3+}$ ion obtained from the crystallographic data.

of the magnetization on decreasing the temperature, with promise for the development of devices in the field of quantum computing [26,27] and high-density memory storage [18,28].

Recently, a second generation of molecular nanomagnets, referred to as single-ion magnets (SIMs) and characterized by a "double-decker" structure entrapping one lanthanide (Ln) trivalent ion, has been reported. These mononuclear lanthanoid complexes are exemplified by phthalocyaninato [29] and by polyoxometalate (POM) complexes [30-32]. Compared with the first generation of nanomagnets, these single-ion magnets have shown to exhibit more pronounced quantum effects making them a very suitable source of molecular spin qubits for quantum computing [31] and, more recently, very promising high-anisotropy-barrier systems with future look to magnetic memories [33,34].

A representative example of $\mathrm{POM}$ complexes is provided by the series $\mathrm{Na}_{9}\left[\mathrm{Ln}\left(\mathrm{W}_{5} \mathrm{O}_{18}\right)_{2}\right] \cdot x \mathrm{H}_{2} \mathrm{O}$. In this paper, we focus on the $\mathrm{Er}^{3+}$ derivative of this family, whose chemical preparation has been already described [30]. The magnetic molecule $\left[\mathrm{Er}\left(\mathrm{W}_{5} \mathrm{O}_{18}\right)_{2}\right]^{9-}\left(\right.$ in short $\left.\mathrm{ErW}_{10}\right)$ consists of a single $\mathrm{Er}^{3+}$ ion with a $J=15 / 2$ ground-state angular momentum sandwiched between two $\left[\mathrm{W}_{5} \mathrm{O}_{18}\right]^{6--}$ POM moieties. In the structure, this molecular anion is surrounded by $\mathrm{Na}^{+}$cations octahedrally coordinated by oxygen atoms and by water molecules. The compound crystallizes in a triclinic structure with $Z=2$ molecules per unit cell. Intermolecular spin-spin interactions are expected to be very weak because $\mathrm{Er}^{3+}-\mathrm{Er}^{3+}$ distances are $\sim 13 \AA$ [32]. The complete formula of the compound studied [30], i.e., $\mathrm{Na}_{9} \mathrm{ErW}_{10} \mathrm{O}_{71} \mathrm{H}_{68}$, shows that the protons that can be detected in an NMR experiment are present only in the water molecules surrounding the $\mathrm{Er}^{3+}$ ion and distributed rather symmetrically around the $\mathrm{Er}^{3+}$ ion as shown in Fig. 1. Thus the protons of the water molecules are a suitable probe of the magnetic static and dynamic properties of the $\mathrm{Er}^{3+}$ ion through the long-range nuclear-electron hyperfine dipolar interaction. The very large difference between the susceptibilities [30-32] along the molecular $z$ axis and those perpendicular to it indicates a strong uniaxial character of the magnetic anisotropy, which is due to the zero-field splitting of $J$ ground state of the lanthanide ion when it is placed in a ligand field (LF). The easy axis for the magnetization is achieved, in this case, by the stabilization of sublevels with a large $\left|J_{z}\right|$ value in a $D_{4 d}$ LF symmetry [35-37]. This large uniaxial anisotropy leads to interesting spin dynamical effects such as the slowing down of the magnetic relaxation (characteristics of the single-molecule magnets, SMMs) and the quantum tunneling of the magnetization at very low temperature [30].

From the above, it is evident that the investigation and modelization of the magnetization dynamics in molecular clusters is crucial for unraveling the physical mechanisms responsible for the observed quantum phenomena and their outlook for future technological applications. On the other hand, to understand in details the spin dynamics of such complexes, the experimental techniques that make use of local probes, like, e.g., NMR, $\mu^{+} \mathrm{SR}$, neutron scattering, and Mossbauer spectroscopy represent an ideal tool. Along this research line, in this paper, the magnetic properties and local spin dynamics of this SIM compound will be investigated by means of NMR and $\mu^{+}$SR techniques. With the former we will study the ${ }^{1} \mathrm{H}$ absorption spectra and the behavior of spin-lattice $\left(T_{1}\right)$ and spin-spin $\left(T_{2}\right)$ relaxation times at three different fields $(B=3.46,1.5,0.43 \mathrm{~T})$ as a function of temperature in the temperature range $1.6 \mathrm{~K}<T<300 \mathrm{~K}$, measured on a powder sample with a Fourier transform (FT) pulse spectrometer. With the latter, we will investigate the magnetic properties and spin dynamics of the sample by analyzing the muon relaxation rates taken, as a function of temperature $(1.5 \mathrm{~K}<$ $T<100 \mathrm{~K}$ ), at two applied longitudinal fields $B=0.05,0.2 \mathrm{~T}$. These measurements have been performed at the Paul Scherrer Institute (PSI) facility in Villigen $(\mathrm{CH})$, on the GPS beam line. We remark that the investigation of the absorption spectra and the dynamics of nuclear and muon probes allows one to access the electronic spin dynamics through the respective hyperfine interaction.

\section{NMR RESULTS}

\section{A. Proton NMR line shape and linewidth}

The NMR line shape was obtained in two different ways: (i) for sufficiently narrow lines such that the intensity of the radio-frequency field was strong enough to irradiate the whole NMR line, the spectra were obtained from the Fourier transform (FT) at half of the echo signal of a standard spin-echo pulse sequence with the $\pi / 2$ pulse in the range $1.7 \mu \mathrm{s}<\pi / 2<$ $3.5 \mu$ s (depending of the magnetic field applied); (ii) for broad lines, the line shape was obtained by plotting the envelope of the FTs of the echo signal by keeping constant the magnetic field and by sweeping the frequency. A few representative ${ }^{1} \mathrm{HNMR}$ spectra, collected at selected temperatures at $B=$ 3.46 and $0.43 \mathrm{~T}$ (inset), are shown in Fig. 2(a) (the spectra at very low temperature at lower field are not displayed, since the signal was poor and the spectra very noisy). The full width at half maximum (FWHM) is plotted as a function of temperature in Fig. 2(b). The data for the very broad spectra below $50 \mathrm{~K}$ 

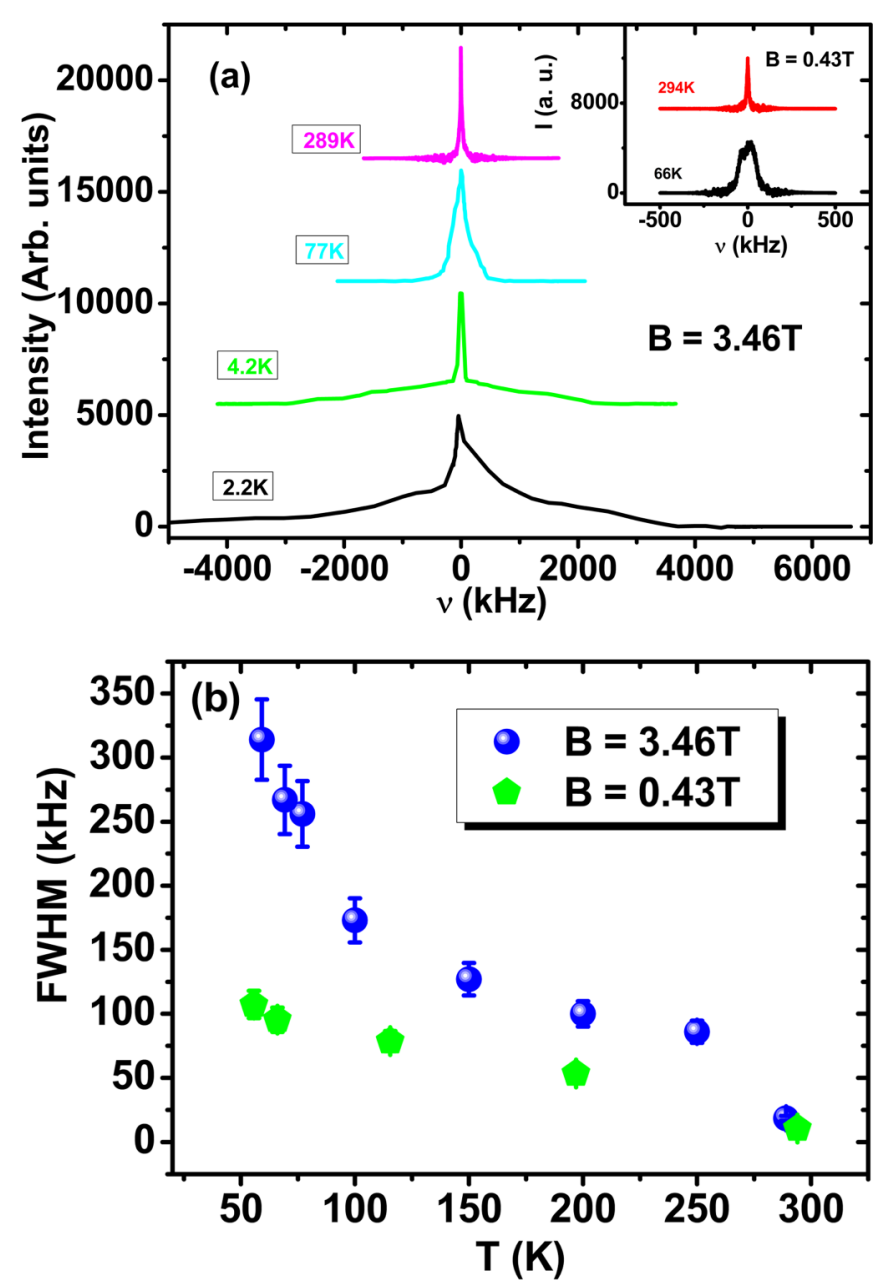

FIG. 2. (a) ${ }^{1} \mathrm{H}$ NMR absorption spectra at selected temperatures for $\mathrm{ErW}_{10}$ at $B=3.46 \mathrm{~T}$ ( $B=0.43 \mathrm{~T}$ in the inset). The very broad spectra at low temperature are obtained by sweeping the frequency as described in the text. (b) FWHM as a function of temperature for $\mathrm{ErW}_{10}$ at $B=3.46$ and $0.43 \mathrm{~T}$. In this plot we report only the data obtained from the Fourier transform of the echo signal, which yields the correct result only if the spectral width of the radio frequency pulse is wider than the NMR width (see text).

are not shown in Fig. 2(b), because the definition of a width is ambiguous; in fact, as seen in Fig. 2(a) for the spectrum at 4.2 and $2.2 \mathrm{~K}$, there is a narrow component associated with the protons far from the magnetic $\mathrm{Er}^{+3}$ and a broad asymmetric line related to the protons, which are subjected to a local hyperfine field. The analysis of the data in Fig. 2 leads to the following qualitative conclusions.

(i) The increase of the linewidth down to about $50-\mathrm{K}$ tracks the Curie-type increase of the magnetic susceptibility [30]. The magnetic field dependence indicates that the broadening is due to the distribution of local hyperfine fields at the proton site due to the interaction with the paramagnetic moment induced by the field on the $\mathrm{Er}^{3+}$ magnetic ion.

(ii) The NMR lines in the paramagnetic region (above $50 \mathrm{~K}$ ) are inhomogeneously broadened without a significant frequency shift of the center from the Larmor frequency (the zero frequency in the graphs) $\omega_{\mathrm{L}}=\gamma B_{0}$. This clearly indicates that the hyperfine interaction between the protons and the magnetic ions is only of dipolar origin; in fact, in a powder sample, the dipolar local field averaged over a large number of nonequivalent protons yields a zero average value.

(iii) Below $50 \mathrm{~K}$, the system no longer behaves as a simple paramagnet; in fact, the effective Curie constant $\chi T$ gradually decreases as a result of depopulation of the highest crystalfield levels [30]. On the other hand, the slowing down of the magnetic fluctuations generates quasistatic local fields at the nuclear site, which, in turn, produce a large broadening of the NMR line. We will refer in the following to this slowing down of the magnetization as spin freezing effect: the spins tend to line up along the anisotropy direction and the experimental detection of this phenomenon depends on the characteristic frequency of the probe. For NMR, the spin freezing occurs when the fluctuation frequency of the single molecule magnetic moment becomes smaller than the precession frequency of the protons in the local field, i.e., in the megahertz $(\mathrm{MHz})$ range.

The NMR line when the local fields become static is no longer proportional to the applied magnetic field and has a structure due to the vector combination of the external field and the local field related to the magnetic moment of the molecule along the anisotropy direction [see Fig. 2(b)]. From the spectrum at $T=2.2 \mathrm{~K}$ [Fig. 2(a)], one can extract the order of magnitude for the distribution of the local fields by evaluating the FWHM of the broad component of the spectrum. One finds a FWHM of about $4.5 \mathrm{MHz}$ corresponding to $\delta B \approx 0.1 \mathrm{~T}$. Similar effects of spin freezing on the NMR line shape have been observed and discussed in low-dimensional molecular magnets [8,16] and in SMMs [38-40].

\section{B. $T_{2}^{-1}$ results and wipe-out effect}

The ${ }^{1} \mathrm{H}$ spin-spin relaxation rate $T_{2}^{-1}$ was measured with a standard Hahn-echo sequence by evaluating the exponential decay of the amplitude of the echo signal as a function of the time of the acquisition of the echo, which is twice the time between the $\pi / 2$ and the $\pi$ pulses of the sequence. The temperature dependence of the proton transverse relaxation rate is shown in Fig. 3. The value of $T_{2}^{-1}$ at room temperature is due to proton nuclear dipole-dipole interactions, it is temperature and magnetic field independent and the order of magnitude is $T_{2}^{-1} \approx 20 \mathrm{~ms}^{-1}$, a value coherent with those of all the molecular magnets [41]. The enhancement of the spin-spin relaxation rate at low temperature is a direct consequence of the slowing down of the magnetic fluctuations as will be discussed further on. We point out here that the evaluation of the $T_{2}^{-1}$ parameter is affected by the instrumental limits of the NMR spectrometer. Since the dead time of the radio frequency receiver is of the order of $\tau_{\mathrm{d}}=10^{-5} \mathrm{~s}$, an echo signal which decays faster than the dead time cannot be detected. Thus when the ${ }^{1} \mathrm{H}$ transverse relaxation time $\mathrm{T}_{2}$ becomes shorter than $\tau_{\mathrm{d}}$, the NMR signal is lost and the $T_{2}$ can no longer be measured. The loss of signal intensity is known as wipe-out effect [38]. It turns out that the progressive loss of signal intensity can be analyzed successfully with a simple model, which captures the main features of the wipe-out effect [38] and allows one to obtain information about the spin dynamics $[38,42]$.

Since the wipe-out effect [38] is due to the divergent behavior of the spin-spin relaxation rate, we assume that the enhancement is related to the zero-frequency components of 


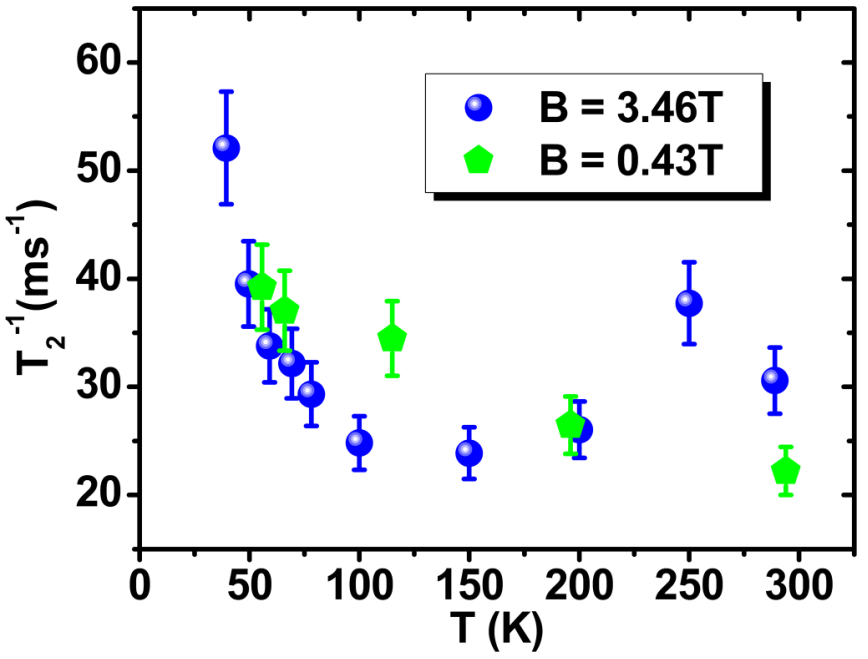

FIG. 3. ${ }^{1} \mathrm{H}$ NMR spin-spin relaxation rate $T_{2}^{-1}$ as a function of temperature for $\mathrm{ErW}_{10}$ at $B=3.46$ and $0.43 \mathrm{~T}$. The increase of the relaxation rate is characteristic of SMMs and it is accompanied by the wipe-out effect [38].

the fluctuations of the local hyperfine fields at the proton site. In the framework of the weak collision, fast motion approximation, the spin-spin relaxation rate can be expressed by the Redfield equation [43]:

$$
T_{2}^{-1}=\gamma_{\mathrm{N}}^{2}\left\langle\delta B_{\mathrm{z}}^{2}\right\rangle \tau(T) \propto \gamma_{\mathrm{N}}^{2} \frac{\left\langle\delta \mu_{\mathrm{e}}^{2}\right\rangle}{r^{6}} \tau(T) .
$$

In Eq. (1), $\left\langle\delta B_{z}^{2}\right\rangle$ is the longitudinal fluctuating field originating from a magnetic moment sitting at a distance $r$ from the ${ }^{1} \mathrm{H}$ spin and $\tau$ is the correlation time (here assumed to be a single dominating correlation time, see next paragraph for a more detailed discussion) where we assumed a spectral density for the fluctuations with Lorenzian form, which reduces simply to $\tau(T)$ in the fast motion limit. We stress that Eq. (1) is no longer valid outside the fast motion limit, namely when $T_{2}$ becomes of the order of $\tau(T)$, a condition equivalent to $\gamma_{\mathrm{N}}\left\langle\delta B_{\mathrm{Z}}\right\rangle \tau \geqslant 1$. Also, we have neglected in Eq. (1) a second term of the form $1 /\left(2 T_{1}\right)$ due to the transverse components of the fluctuating local field [43]. The idea underlying the model for the analysis of the wipe-out effect [38] is that on decreasing the temperature the correlation time $\tau$ becomes longer and longer and, as a consequence, the spin-spin relaxation time shortens until it reaches the limiting value $\tau_{\mathrm{d}}$ below which the signal cannot be detected in our experimental setup. Being the dipolar hyperfine field, and thus $T_{2}$, dependent on the distance $r$ between the magnetic ions and the protons sites, $\tau_{\mathrm{d}}$ is reached by all the protons of the sample but with the ones closer to the magnetic centers wiped-out first, leading to a gradual loss of proton NMR signal. We summarize now the model adopted in Ref. [38]. It is assumed a central magnetic ion surrounded by a sphere containing a number $n_{0}$ of protons in each molecule at various distances, up to a maximum value $R^{*}$. The density of ${ }^{1} \mathrm{H}$ nuclei in each molecule is defined by the equation

$$
\rho=\frac{n_{0}}{\frac{4}{3} \pi\left(R^{*}\right)^{3}} .
$$

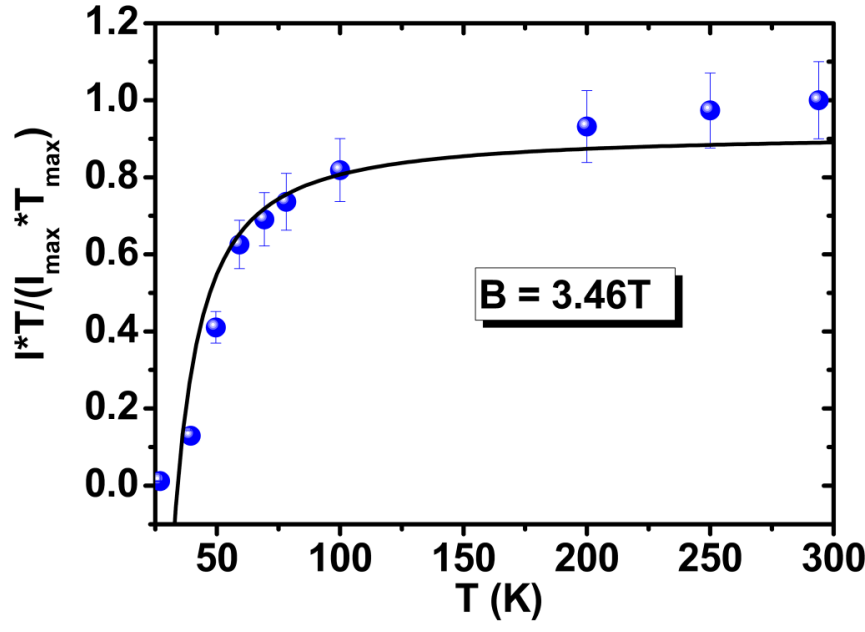

FIG. 4. ${ }^{1} \mathrm{H}$ NMR normalized signal intensity multiplied by $T$ and plotted vs T for $\operatorname{ErW}_{10}$ at $B=3.46 \mathrm{~T}$. The solid line is the best-fit to the experimental data following Eq. (4) in the text.

At each specific temperature and value of the correlation time, there are some protons having $T_{2}^{-1}>10^{5} \mathrm{~s}^{-1}$ and located within an ideal sphere whose critical radius $r_{c}$ depends on the temperature. These ${ }^{1} \mathrm{H}$ nuclei do not contribute to the detected NMR signal. On the other hand, the number $n(T)$ of protons outside this sphere and contributing to the signal intensity can be quantified by the equation

$$
n(T)=n_{0}\left[1-\left(\frac{r_{c}}{R^{*}}\right)^{3}\right]
$$

The value of the critical radius in Eq. (3) can be obtained by setting $T_{2}$ equal to $\tau_{\mathrm{d}}$ in Eq. (1) and solving for $r_{c}$. By substituting in Eq. (3) the critical radius, one has for the fraction of protons contributing to the NMR signal,

$$
\frac{n(T)}{n_{0}}=1-\frac{\gamma_{N} \sqrt{\delta \mu_{\mathrm{e}}^{2}} \sqrt{\tau_{d}}}{R^{* 3}} \sqrt{\tau(T)} .
$$

In order to use Eq. (4) to fit the data, we adopt an Arrheniuslike behavior for the correlation time $\tau=\tau_{0} \exp (\Delta / T)$, where $\tau_{0}$ is a pre-exponential factor and $\Delta$ an activation energy.

The NMR signal at each temperature was obtained from the exponential decay of the plot of the integral of the echo signal intensity as a function of twice the time between the two pulses of the Hahn sequence and extrapolating back the echo intensity at $\tau=0$ with the assumption that the echo signal follows a single-exponential decay over the whole time range.

The results are shown in Fig. 4 and fitted with Eq. (4) by choosing as fitting parameters $\left(\left\langle\delta \mu_{\mathrm{e}}^{2}\right\rangle\right)^{1 / 2} / R^{* 3}=557 \mathrm{G}$, $\tau_{0}=3 \times 10^{-12} \mathrm{rad}^{-1} \mathrm{~s}$, and $\Delta=170 \mathrm{~K}$. The magnitude of the parameter $\left(\left\langle\delta \mu_{\mathrm{e}}^{2}\right\rangle\right)^{1 / 2} / R^{* 3}$ expresses the local hyperfine field felt by the proton site: the value obtained is comparable with the local field which broadens the NMR line at low temperature as shown in Fig. 2 and with measurements in other molecular magnets [38,44]. A check of the consistency of our model can be made by estimating the maximum radius $R^{*}$ from the value of $\left(\left\langle\delta \mu_{\mathrm{e}}^{2}\right\rangle\right)^{1 / 2} / R^{* 3}$ obtained from the fit with Eq. (4). 


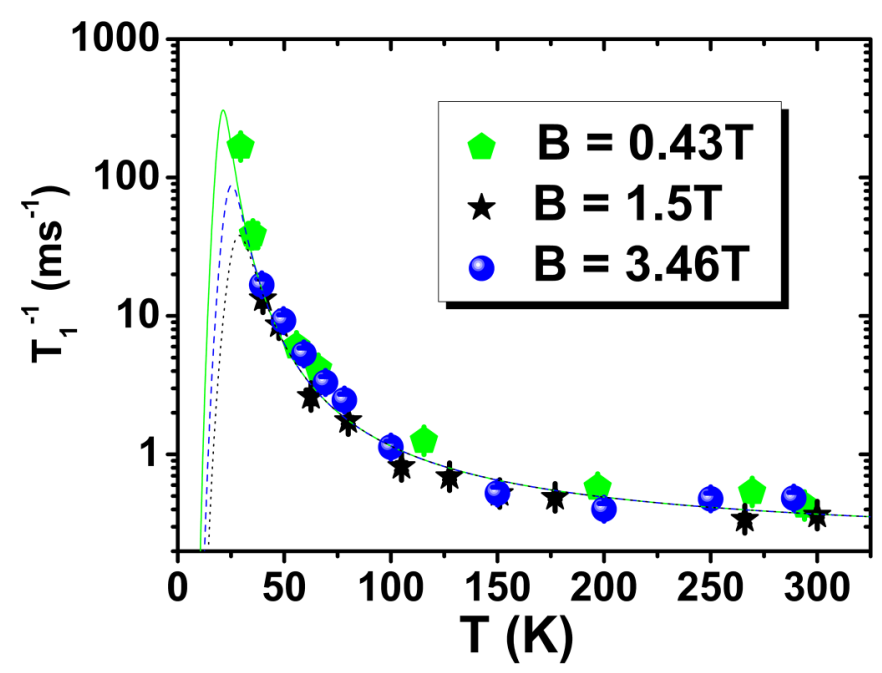

FIG. 5. ${ }^{1} \mathrm{H}$ NMR spin-lattice relaxation rate $T_{1}^{-1}$ as a function of temperature for $\mathrm{ErW}_{10}$ at $B=3.46,1.5$, and $0.43 \mathrm{~T}$. The peak expected for NSLR and described by Eq. (5) cannot be reached by the experimental points because of the wipe-out effect described in the previous paragraph. The lines are best-fits of the experimental data according to Eq. (5) with the fitting parameters summarized in the text.

By assuming for the fluctuating moment the value of the moment of the $\mathrm{Er}^{3+}$ ion in the $J=15 / 2$ ground state, one obtains $R^{*}=5.5 \AA$. This value compares well with the average distance of the protons in the water molecules from the central $\mathrm{Er}^{3+}$ ion. In fact, the oxygen atoms of the water molecules (see Fig. 1) are located at a distance from the $\mathrm{Er}^{3+}$ ion, which ranges from a minimum of $4 \AA$ to a maximum of $12 \AA$. The pre-exponential factor and the activation energy were chosen to be the same as the ones used in the fit of the NMR relaxation rates as shown in Sec. III.

\section{Proton $T_{1}^{-1}$ results}

The proton spin-lattice relaxation rate $T_{1}^{-1}$ was measured by applying a saturation recovery sequence of radio-frequency pulses and monitoring the recovery of the nuclear magnetization towards thermal equilibrium. The recovery curve of the magnetization was found to be biexponential for all the magnetic field applied and in the entire range of temperatures investigated. At high temperatures the weight of the longer component was less than 5\% and thus it was disregarded and we focused only on the first component of the exponential decay. In the temperature region where the NMR signal decreases as a result of the wipe-out effect [38], the relative weight of the long decaying component increases but we kept neglecting it since it arises from protons, which are far away from the $\mathrm{Er}^{3+}$ magnetic moments and thus do not convey any information about the spin dynamics. The results of proton $T_{1}^{-1}$ are shown in Fig. 5 as a function of temperature and for different applied magnetic fields. The measurements could not be extended to low temperature because of the wipe-out effect [38] described in the previous section. In order to analyze the results, we adopt an expression for the spin-lattice relaxation, which has proved to describe the behavior of $T_{1}^{-1}$ in molecular magnets
[42,44-47]. The expression is derived from the general formula of Moriya for nuclear relaxation in paramagnets [45] under the simplifying assumption that the relaxation is driven by the fluctuations of the local hyperfine field whose spectral density is a simple Lorenzian characterized by a single correlation time. Then, the expression of the nuclear spin-lattice relaxation rate (NSLR) is

$$
T_{1}^{-1}=\frac{A^{\prime}\left(\omega_{c}(T)\right)}{\omega_{c}^{2}(T)+\omega_{L}^{2}}=A \chi T \frac{\omega_{c}(T)}{\omega_{c}^{2}(T)+\omega_{L}^{2}},
$$

where $A \chi T$ is the mean square value of the fluctuations of the transverse hyperfine field at nuclear sites, $\omega_{\mathrm{c}}(T)=2 \pi / \tau(T)$ is the correlation frequency, $\omega_{\mathrm{L}}$ is the Larmor frequency, and $\chi$ is the uniform magnetic susceptibility expressed in emu/mol.

This model predicts a maximum in the NSLR as a function of temperature when the correlation frequency of the fluctuations becomes of the order of the Larmor frequency. As a matter of fact, such a maximum has been observed in most of the molecular nanomagnets previously investigated [42,46-48]. In our case, as shown in Fig. 5, the measurements cannot be extended into the low temperature region, where the maximum in $T_{1}^{-1}$ is expected to occur, because of the loss of NMR signal, i.e., the wipe-out effect [38] described in the previous section. Nevertheless, one can obtain partial information about the spin dynamics by fitting the high-temperature region where the $T_{1}^{-1}$ becomes magnetic field independent (see Fig. 5). The high-temperature region corresponds to the so-called fast motion regime, namely $\omega_{\mathrm{c}} \gg \omega_{\mathrm{L}}$, where Eq. (5) becomes field independent. Thus the fits in Fig. 5 obtained from Eq. (5) by assuming $\left(\omega_{\mathrm{c}} / 2 \pi\right)^{-1}=\tau=\tau_{0} \exp (\Delta / T)$ cannot determine separately the two parameters $\tau_{0}$ and $A$. The fitting parameters $A \chi T=7 \times 10^{13} \mathrm{~s}^{-2} \mathrm{rad}^{2} \mathrm{emu} \mathrm{K} \mathrm{mol}-1, \tau_{0}=3 \times$ $10^{-12} \mathrm{~s} \mathrm{rad}^{-1}$, and $\Delta=170 \mathrm{~K}$, correspond to the longest $\tau_{0}$ consistent with the position of the peak and consequently the smaller value of $A$ and consistent with the ones found for proton NMR in most molecular magnets [42,48]. The term $\chi T$ corresponds to an effective Curie constant and is estimated to be $C=11.5 \mathrm{emu} \mathrm{K} \mathrm{mol}^{-1}$ for the paramagnetic $\mathrm{Er}^{3+}$ in the $J=15 / 2$ ground state [30]. It is worth to note that (i) the Arrhenius formula $\tau=\tau_{0} \exp (\Delta / T)$ has been used on the basis of previous theoretical predictions and experimental works [46-50]; (ii) the value $\Delta=170 \mathrm{~K}$ represents a high-temperature average activation energy, which is strictly connected to the structure of the energy levels. More details about the link among the energy levels and the behavior of $T_{1}^{-1}$ in the different temperature regimes will be given after the presentation of the $\mu^{+}$SR results in the next section.

It should be remarked that alternative approaches can be used to describe the nuclear (muon) spin-lattice relaxation in molecular magnets. In $\mathrm{Mn}_{12}$, the NMR and $\mu^{+} \mathrm{SR}$ relaxation data at low temperature were explained in terms of energy exchange between the nucleus (muon) and the thermal fluctuations of the magnetization in the ground state associated with spin-phonon interactions [51]. In $\mathrm{LiY}_{0.998} \mathrm{Ho}_{0.002} \mathrm{~F}_{4}$, the nuclear relaxation was interpreted in terms of quasielastic scattering with the lifetime broadened magnetic energy levels of the $\mathrm{Ho}^{3+}$ magnetic ion [52]. In both cases, the expression for the nuclear (muon) spin-lattice relaxation is similar to the one adopted here although the meaning of the parameters involved 


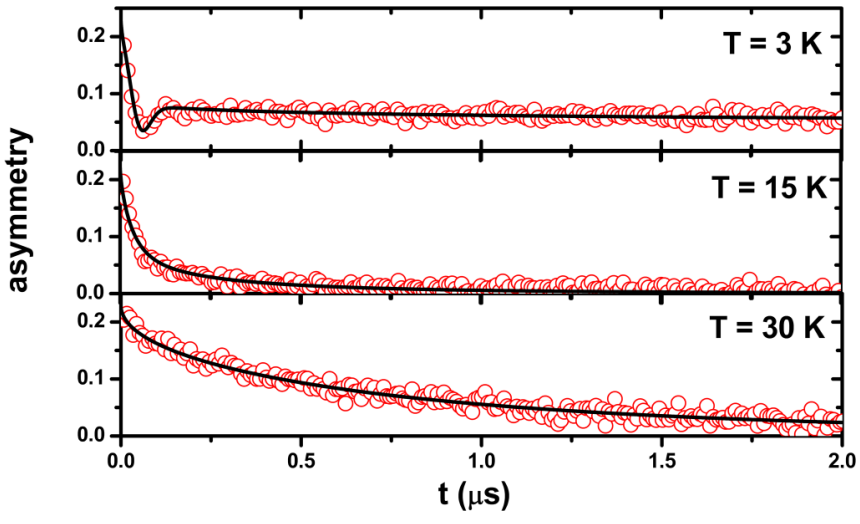

FIG. 6. Time dependence of the muon asymmetry at three representative temperatures in a longitudinal magnetic field of $0.05 \mathrm{~T}$. The black lines represent the best fits of the decay following the model described in the text.

may be different as it will be further discussed in the last paragraph.

As a consequence of the wipe-out effect [38], the maximum expected in the proton NMR spin-lattice relaxation rate cannot be observed. Hence, we performed $\mu^{+}$SR measurements. With this technique one can measure very short muon spin-lattice relaxation times, which occur at low temperatures when the fluctuations of the magnetization become slow, i.e., of the order of the inverse of the muon Larmor frequency.

\section{III. $\mu^{+}$SR RESULTS}

\section{A. Asymmetry decay and internal magnetic fields}

The $\mu^{+}$SR relaxation rates were measured as a function of temperature in longitudinal magnetic fields of 0.05 and $0.2 \mathrm{~T}$. Some representative curves of the muon decay asymmetry are shown in Fig. 6 for $B=0.05 \mathrm{~T}$. Below approximately $15 \mathrm{~K}$, a peculiar dip in the muon asymmetry is observed (see Fig. 6), as expected for a distribution of quasistatic magnetic fields at the muon stopping site, which causes a coherent precession of the spins of those muons with a component of their spin polarization perpendicular to the local field.

The muon asymmetry $A_{\mu}(t)$ was fitted in the entire temperature range for both applied longitudinal magnetic fields, by means of a function expressed as a sum of three relaxing components. Thus the fitting functions have been written as follows:

For $B=0.05 \mathrm{~T}$ :

$$
\begin{aligned}
A_{\mu}(t)= & A_{\text {fast }} \exp \left(-\lambda_{\text {fast }} t\right)^{\beta 11}+A_{\text {interm }} G_{\mathrm{KT}}\left(t, \Delta_{\mathrm{KT}}\right) \\
& \times \exp \left(-\lambda_{\text {interm }} t\right)^{\beta 12}+A_{\text {slow }} \exp \left(-\lambda_{\text {slow }} t\right)^{\beta 13}, \\
& \quad \text { for } T<15 \mathrm{~K},
\end{aligned}
$$

while for $T>15 \mathrm{~K}$, the second term is replaced by $A_{\text {interm }} \exp \left(-\lambda_{\text {interm }} \mathrm{t}\right)^{\beta 12}$.

For $B=0.2 \mathrm{~T}$ (all temperatures):

$$
\begin{aligned}
A_{\mu}(t)= & A_{\text {fast }} \exp \left(-\lambda_{\text {fast }} t\right)^{\beta 21}+A_{\text {interm }} \exp \left(-\lambda_{\text {interm }} t\right)^{\beta 22} \\
& +A_{\text {slow }} \exp \left(-\lambda_{\text {slow }} t\right)^{\beta 23}
\end{aligned}
$$

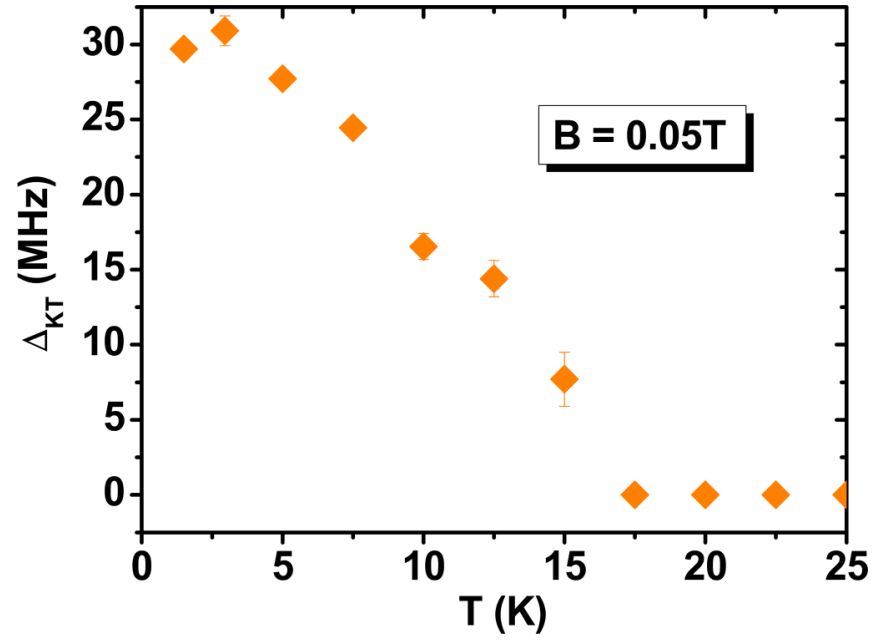

FIG. 7. Temperature dependence of the precession frequencies showing the onset of quasistatic local magnetic fields below $15 \mathrm{~K}$.

In the above expressions, $\beta_{\mathrm{ii}}$ are stretched exponents, $A_{\text {fast }}=5$, $A_{\text {interm }}=15.8, \mathrm{~A}_{\text {slow }}=2.7$, and $\lambda_{\text {fast }}, \lambda_{\text {interm }}$, and $\lambda_{\text {slow }}$ are the weights and the correspondent muon longitudinal relaxation rates of three inequivalent groups of muons implanted at different distances of the magnetic core: the fast component corresponds to the muons nearest to the core, the intermediate one to muons in intermediate position, and the slowest one to muons far from the magnetic ions. Additionally, it should be noted that (i) the fast component relaxation rate $\lambda_{\text {fast }}$ is not reported (and discussed) because its values fall, especially for $T \leqslant 30 \mathrm{~K}$, within the deadtime of the $\mu^{+}$SR instrument; (ii) the slowly relaxing component has a very low relative weight and so the corresponding $\lambda_{\text {slow }}(B, T)$ curves will not be considered in the following due to possible low statistical significance; it should be, however, noted that the $\lambda_{\text {slow }}$ versus $T$ behavior at the two applied fields (data not reported) is qualitatively similar to the one of $\lambda_{\text {interm versus }} T$; (iii) the stretched exponents $\beta_{\text {ii }}$ assume values in the range $0.5<\beta<1$ over the whole $T$ range for both applied fields; (iv) as shown above, for $T<15 \mathrm{~K}$, the "intermediate" relaxing component constitutes a Kubo-Toyabe function $G_{\mathrm{KT}}\left(t, \Delta_{\mathrm{KT}}\right)$ [53] multiplied by a stretched exponential. The "intermediate" component at short times accounts for the behavior of the polarization of muons feeling a distribution of magnetic local fields (of Gaussian or Lorentzian shape, with FWHM equal to $\Delta_{\mathrm{KT}}$ ) due to electronic spins fluctuating with a frequency smaller than the muon Larmor frequency, and its minimum measures the width $\Delta_{\mathrm{KT}}$ of the distribution of the local fields at the muon site; at long times, the stretched exponential behavior of the $A_{\text {interm }}(t)$ tail is due to a slow dynamics of the local fields.

From the analysis of the polarization curves, we extracted the distribution of the local field $\Delta_{\mathrm{KT}}$ and the muon relaxation rate $\lambda_{\text {interm }}$. As can be seen from Fig. 7 , there is a progressive onset of quasistatic local fields starting at about $15 \mathrm{~K}$. The width of the distribution reaches, at the lowest temperature measured, a value of about $\Delta_{\mathrm{KT}}=30 \mathrm{MHz}$ corresponding to $\delta B \approx 0.22 \mathrm{~T}$. The onset of quasistatic local fields at low temperature is indicative of a dynamical spin freezing of the individual moments of the SMM along the magnetic anisotropy 


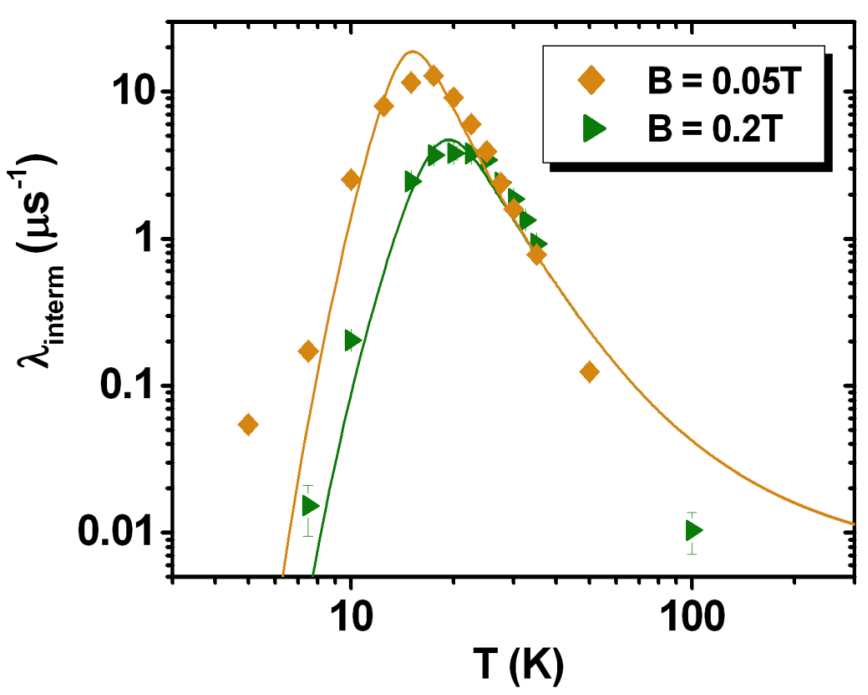

FIG. 8. Muon longitudinal relaxation rates plotted as a function of temperature in a longitudinal magnetic field of 0.05 and $0.2 \mathrm{~T}$. The two lines correspond to the fit according to Eqs. (6) and (7) with parameters discussed in the text.

axis arising from the zero field splitting of the $\mathrm{Er}^{3+} J$ ground state in a ligand field [30] in agreement with the NMR spectra at low temperature.

\section{B. Muon spin-lattice relaxation rate}

Any fluctuation in the magnitude of the internal local fields results in a decay of the muon asymmetry function described by the relaxation parameter $\lambda$, which is the muon equivalent of the proton spin-lattice relaxation rate $T_{1}^{-1}$ described in the previous section.

The results for the temperature dependence of the muon spin-lattice relaxation (SLR) $\lambda$ are shown in Fig. 8. As can be seen, for the 0.05-T longitudinal magnetic field, i.e., $v_{\mu}=$ 6.7769 MHz, there is a maximum around $18 \mathrm{~K}$ corresponding to fluctuations of the magnetization in the frequency range of the muon Larmor frequency. At 0.2-T longitudinal applied field, the muon Larmor frequency is higher and consequently the maximum appears at higher temperature.

If one fits the data in Fig. 8 by using Eq. (5) and the same parameters used to fit the proton NMR relaxation or even different parameters, the fit is only qualitative. In fact, Eq. (5) is based on a phenomenological model where all fluctuations of the magnetization are described by a single correlation time and the corresponding effective average activation energy. Such an oversimplified model can work on a limited temperature range as for the NMR data in Figs. 4 and 5. However, a quantitative fit over the whole temperature range requires a more detailed model in which one takes into account the different magnetic energy levels of the SMM. We will adopt here the model that has been successful in describing the thermal fluctuations in the magnetic ground state of the molecular cluster $\mathrm{Mn}_{12}$ [51].

For the $\mathrm{ErW}_{10}$ system investigated here, the levels are the crystal field split $M_{J}$ levels of the $J=15 / 2 \mathrm{Er}^{3+}$ magnetic ground state [30]. According to a recent report [54], the ground state is $\mathrm{M}_{\mathrm{J}}= \pm 13 / 2$, the first excited state is $M_{J}= \pm 1 / 2$

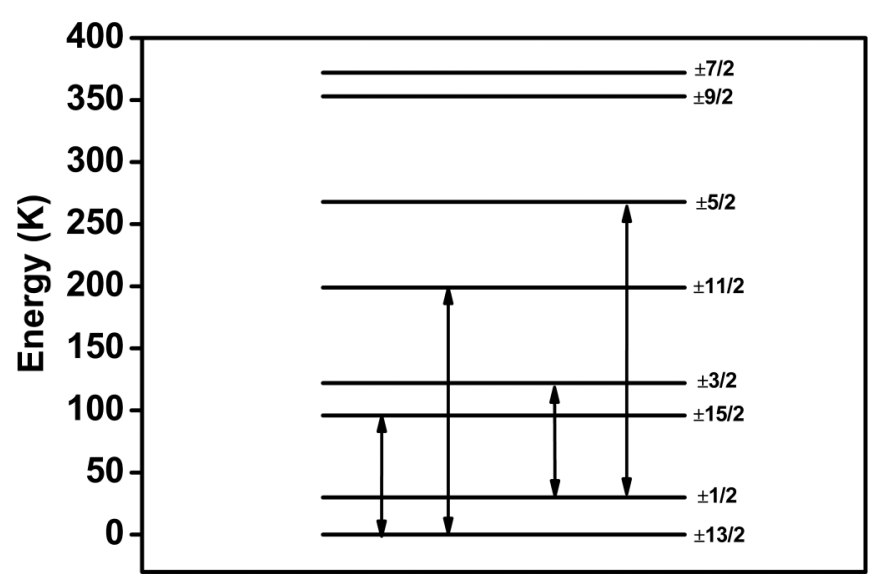

FIG. 9. Scheme of the energy levels for $J=15 / 2$ erbium in ErW $_{10}$ complex according to Ref. [54].

at $30 \mathrm{~K}$ above the ground state and higher excited states are found at 96, 122, 199, and $268 \mathrm{~K}$. A complete scheme of the energy levels is shown in Fig. 9. The vertical arrows indicate the allowed spin-phonon transitions from the ground state and first excited state.

Muon (nuclear) spin-lattice relaxation involves the exchange of a small quantum of Zeeman energy with the "lattice" and is thus possible only because a lifetime broadening of the magnetic energy levels of the $\mathrm{Er}^{3+}$ ion is present. In the framework of the weak collision approximation $[43,45]$, the above "scattering" process can be described in terms of the correlation function of the transverse component of the time-dependent hyperfine field at the muon (nuclear) site. In our case, the hyperfine interaction is the muon (nuclear) dipolar interaction with the magnetic moment of the $\mathrm{Er}^{3+}$ ion and the time dependence comes from the changes of orientation of the $\mathrm{Er}^{3+}$ magnetic moment due to transitions among the different $M_{J}$ levels (see Fig. 9). Following Ref. [51], we assume an exponential correlation function for the hyperfine field with correlation frequency $\omega_{M j}$ corresponding to a Lorentzian broadening of the $M_{J}$ sublevel. We can then write

$$
\frac{1}{T_{1}}(\lambda)=\frac{A}{Z} \sum_{M_{J}} \frac{\exp \left(\frac{E_{M_{J}}}{k_{B} T}\right) \omega_{M_{J}}}{\omega_{M_{J}}^{2}+\omega_{L}^{2}},
$$

where $Z$ is the partition function and the sum is over the few low-lying magnetic energy levels. The correlation frequency $\omega_{M j}$ is the reciprocal of the lifetime broadening of the corresponding energy level and is expressed [51] as the sum of spin-phonon transition probabilities, which will be assumed to be given by an exponential form with an activation barrier given by the separation of the magnetic energy levels:

$$
\omega_{M_{J}}=\omega_{0} \exp \left(-\frac{\Delta_{\mathrm{M}_{\mathrm{J}}}}{k_{B} T}\right)+\omega_{0}^{\prime} \exp \left(-\frac{\Delta_{\mathrm{M}_{J^{\prime}}}}{k_{B} T}\right)+\ldots
$$

The experimental results in Fig. 8 are fitted by using Eqs. (6) and (7) by considering only the ground-state level $\pm 13 / 2$ and the first excited state $\pm 1 / 2$ separated by $30 \mathrm{~K}$. By using the energy level scheme and the energy separation given in Fig. 9, we express the correlation frequency in terms of the energy separation whereby the ground-state $\pm 13 / 2$ is connected by 


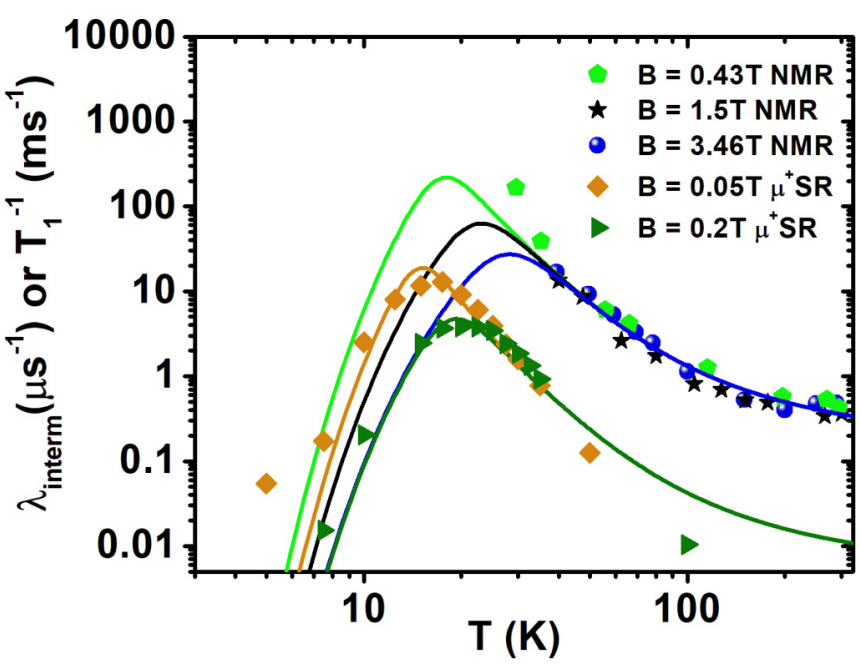

FIG. 10. Comparison of the spin-lattice relaxation rates for proton NMR and for $\mu^{+}$SR at different magnetic fields. The lines are fitting curves according to Eqs. (6) and (7) with the set of parameters discussed in the text.

the allowed selection rule to the excited state $\pm 15 / 2$ at $96 \mathrm{~K}$ and the other excited state $\pm 11 / 2$ at $199 \mathrm{~K}$, while the first excited state $\pm 1 / 2$ is connected to the state $\pm 3 / 2$ at $92 \mathrm{~K}$ and to the state $\pm 15 / 2$ at $238 \mathrm{~K}$.

The fit is shown by the two curves in Fig. 8. By using the energy separations given in literature, there are only two fitting parameters, i.e., $A$, which is the amplitude of the hyperfine interaction, and $\omega_{0}$, which is the strength of the spin-phonon interaction, assumed to be proportional to the cube of the energy separation of the transition considered [51].

Thus $\omega_{0}$ ' is assumed to be the same as $\omega_{0}$ simply rescaled as the cube of the energy separation. The fit (see Fig. 8) is good by assuming $A=1.6 \times 10^{15} \mathrm{~s}^{-2} \mathrm{rad}^{2} \mathrm{emu} \mathrm{K} \mathrm{mol}^{-1}$ and $\omega_{0}=2 \times 10^{10} \mathrm{~Hz}$ rad. The deviation of the relaxation data $\lambda$ from the fitting curve at very low temperature is due to the fact that when the spin freezing is reached, the weak collision approach, which Eqs. (5) and (6) rely on, is no longer valid. In Fig. 10, we show the comparison of the NMR and the $\mu^{+}$SR relaxation data over the whole temperature and magnetic field range. It is remarkable that both sets of data seem to be reproduced reasonably well by Eqs. (6) and (7) with the same set of parameters used to fit the $\mu^{+} \mathrm{SR}$ except for the strength of the interaction $A=5 \times 10^{13} \mathrm{~s}^{-2} \mathrm{rad}^{2} \mathrm{emu} \mathrm{K} \mathrm{mol}^{-1}$, which is less for the protons due to the smaller gyromagnetic ratio with respect to the muons. The strength of the average square fluctuating hyperfine field at the proton (muon) site determined by the constant $A$ in the longitudinal rate, Eq. (6), resulted in $A_{\text {proton }}=5 \times 10^{13} \mathrm{~s}^{-2} \mathrm{rad}^{2} \mathrm{emu} \mathrm{K} \mathrm{mol}{ }^{-1}$ and $\mathrm{A}_{\text {muon }}=160 \times 10^{13} \mathrm{~s}^{-2} \mathrm{rad}^{2} \mathrm{emu} \mathrm{K} \mathrm{mol}^{-1}$ leading to $\left(A_{\text {muon }} \gamma_{\mathrm{N}}^{2} / A_{\text {proton }} \gamma_{\mu}^{2}\right)^{1 / 2}=3.2$. Since, for dipolar interaction, $A$ should scale with the square of the gyromagnetic ratio $\gamma$, the value obtained indicates that the implanted muons must be, on the average, closer to the magnetic center than the protons. This conclusion is in agreement with what found from the static measurements discussed above.

\section{SUMMARY AND CONCLUSIONS}

We investigated the spin dynamics of the SIM system $\left[\mathrm{Er}\left(\mathrm{W}_{5} \mathrm{O}_{18}\right)_{2}\right]^{9-}$ in a wide temperature range for different applied magnetic fields, by means of NMR and $\mu^{+}$SR spectroscopy and relaxation. From experimental measurements, the NMR absorption spectra and the longitudinal relaxation rates $1 / T_{1}$ (NMR) and $\lambda\left(\mu^{+} \mathrm{SR}\right)$ versus $T$ and $B$ have been measured, thus giving information on static and dynamic local magnetic properties of the compound.

As concerns the static properties, both NMR broadening and $\mu^{+}$SR asymmetry decay functions indicate the onset of local quasistatic fields below $10 \mathrm{~K}$. Their order of magnitude reaches, at the lowest temperature, about $0.1 \mathrm{~T}$ for NMR (see Fig. 2) and $0.2 \mathrm{~T}$ for $\mu^{+} \mathrm{SR}$ (see Fig. 7). These local fields are of the correct order of magnitude for proton (muon) nuclear-electron dipolar interaction, whereby the larger value for muons should be due to shorter distances, on average, of the implanted muons from the $\mathrm{Er}^{3+}$ moment than the protons. The broadening of the ${ }^{1} \mathrm{H}$ (muon) static local field distribution as a function of temperature is very gradual, because the condensation of the system in the ground state occurs slowly when temperature is decreased and the higher energy levels are depopulated.

Regarding the dynamical magnetic properties, both NMR and $\mu^{+}$SR spin-lattice relaxation indicate the slowing down of the fluctuations of the magnetization with an Arrhenius-type law on approaching the spin freezing temperature, defined as the temperature below which the fluctuation time of the electronic spins becomes firstly of the order of, and subsequently progressively longer than, the inverse of the Larmor precession frequency of the proton (muon). The fit of the proton NMR and $\mu^{+}$SR longitudinal relaxation rates vs $\mathrm{T}$ at higher temperatures can be described reasonably well by using a thermally activated law with a single correlation time: $\tau(\mathrm{T})=$ $3 \times 10^{-12} \exp (170 / T)$. However, in order to fit the relaxation data over the whole temperature and field range, one has to use a more detailed model, which takes into account the $\mathrm{Er}^{3+}$ energy levels and their separation. We have shown in Figs. 8 and 10 that by using the model in Ref. [51] and the energy level separation given in Ref. [54], there is a good agreement for both proton NMR and $\mu^{+} \mathrm{SR}$ relaxation. This is a confirmation of the validity of the energy level scheme in Ref. [54] with a broadening of the levels due to spin-phonon transitions among the $\mathrm{Er}^{3+} M_{J}$ sublevels. It should be remarked that the application of an external magnetic field is expected to mix different $\mathrm{M}_{\mathrm{J}}$ substates and thus spin-phonon transitions with $\Delta M_{J}$ greater than $\pm 1, \pm 2$ could contribute. The calculation of this second-order contribution is a complex theoretical endeavor and although it may improve somewhat the theoretical fits, it would not provide additional information about spin-phonon transition probabilities in view of the uncertainty in the experimental results. One should mention that in the very low-temperature study of the susceptibility measurements [31], it was found that the magnetization-relaxation process is governed by an Arrhenius-type law with an activation energy $\Delta=55.2 \mathrm{~K}$. Furthermore, in Ref. [32], it was shown that at very low temperature $(4 \mathrm{~K})$ the magnetization of the $\mathrm{Er}^{3+}$ ion can only reverse its spin via quantum tunneling processes. In 
the tunneling regime, the relaxation time becomes temperature independent. It would be interesting to investigate the tunneling regime by NMR $\left(\mu^{+} \mathrm{SR}\right)$ where the spin-lattice relaxation is driven by a strong collision and gives a direct measurement of the tunneling frequency [55]. However, a warning has to be given for what concerns the eventual flattening of the $\mu^{+}$SR relaxation rate $\lambda(\mathrm{T})$, due to the effect of tunnelling: this effect have been evidenced in more than one paper [49] on very different molecular clusters and recently shown in a more complete way in Ref. [50], but its physical origin is still subject of debate and not attributable with certainty to quantum effects.

\section{ACKNOWLEDGMENTS}

This work has been supported by the European Commission (COST Action 15128 on Molecular Spintronics), the Spanish MINECO (Project MAT2017-89993-R and Unit of Excellence María de Maeztu MDM-2015-0538) and the Generalitat Valenciana (Prometeo program). The projects EU-FP6-NoE MAGMANET and the FIRB project No. RBFR12RPD1 of the Italian Ministry of Education and Research, are acknowledged for partly funding this research. The authors are grateful to P. Carretta and B. Z. Malkin for useful discussions and suggestions. R. De Renzi and G. Allodi are also gratefully acknowledged.
[1] D. Gatteschi, R. Sessoli, and J. Villain, Molecular Nanomagnets (Oxford University Press, New York, 2006).

[2] O. Kahn, Molecular Magnetism (VCH, Berlin, 1990).

[3] R. J. Glauber, J. Math. Phys. 4, 294 (1963).

[4] A. Caneschi, D. Gatteschi, N. Lalioti, C. Sangregorio, R. Sessoli, G. Venturi, A. Vindigni, A. Rettori, M. G. Pini, and M. A. Novak, Angew. Chem. Int. Ed. 40, 1760 (2001).

[5] A. Caneschi, D. Gatteschi, N. Lalioti, C. Sangregorio, R. Sessoli, G. Venturi, A. Vindigni, A. Rettori, M. G. Pini, and M. A. Novak, Europhys. Lett. 58, 771 (2002).

[6] M. Mariani, S. Aldrovandi, M. Corti, J. Lago, A. Lascialfari, E. Micotti, A. Rettori, F. Cinti, A. Amato, C. Baines, L. Bogani, A. Caneschi, S. P. Cottrell, and D. Gatteschi, Inorganica Chimica Acta 360, 3903 (2007).

[7] M. G. Pini and A. Rettori, Phys. Rev. B 76, 069903(E) (2007).

[8] M. Mariani, S. Aldrovandi, M. Corti, A. Lascialfari, L. Bogani, A. Caneschi, and R. Sessoli, Inorganica Chimica Acta 361, 4107 (2008).

[9] M. G. Pini, A. Rettori, L. Bogani, A. Lascialfari, M. Mariani, A. Caneschi, and R. Sessoli, Phys. Rev. B 84, 094444 (2011).

[10] J. Villain, J. Phys. (France) 35, 27 (1974).

[11] J. Villain, J. Phys. (France) 38, 385 (1977).

[12] J. Villain in Chiral Order in Helimagnets, Proceedings of the 13th IUPAP Conference on Statistical Physics, edited by C. Weil, D. Cabib, C. G. Kuper, and I. Riess (Ann. Isr. Phys. Soc., 1978), Vol. 2, p. 565.

[13] M. Affronte, A. Caneschi, C. Cucci, D. Gatteschi, J. C. Lasjaunias, C. Paulsen, M. G. Pini, A. Rettori, and R. Sessoli, Phys. Rev. B 59, 6282 (1999).

[14] A. Lascialfari, R. Ullu, M. Affronte, F.Cinti, A. Caneschi, D. Gatteschi, D. Rovai, M. G. Pini, and A. Rettori, Phys. Rev. B 67, 224408 (2003).

[15] F. Cinti, M. Affronte, A. Lascialfari, M. Barucci, E. Olivieri, E. Pasca, A. Rettori, L. Risegari, G. Ventura, M. G. Pini, A. Cuccoli, T. Roscilde, A. Caneschi, D. Gatteschi, and D. Rovai, Polyhedron 24, 2568 (2005).

[16] M. Mariani, A. Lascialfari, A. Caneschi, L. Ammannato, D. Gatteschi, A. Rettori, M. G. Pini, C. Cucci, and F. Borsa, Phys. Rev. B 93, 134410 (2016).

[17] F. Cinti, A. Rettori, M. G. Pini, M. Mariani, E. Micotti, A. Lascialfari, N. Papinutto, A. Amato, A. Caneschi, D. Gatteschi, and M. Affronte, Phys. Rev. Lett. 100, 057203 (2008).

[18] R. Sessoli, D. Gatteschi, A. Caneschi, and M. A. Novak, Nature (London) 365, 141 (1993).
[19] B. Barbara, W. Wernsdorfer, L. C. Sampaio, J. C. Park, C. Paulsen, M. A. Novak, R. Ferrè, D. Mailly, R. Sessoli, A. Caneschi, K. Hasselbach, A. Benoit, and L. Thomam, J. Magn. Magn. Mater. 140-144, 1825 (1995).

[20] L. Thomas, F. Lionti, R. Ballou, D. Gatteschi, R. Sessoli, and B. Barbara, Nature (London) 383, 145 (1996).

[21] J. R. Friedman, M. P. Sarachik, J. Tejada, and R. Ziolo, Phys. Rev. Lett. 76, 3830 (1996).

[22] A. A. Mukhin, V. D. Travkin, A. K. Zvezdin, S. P. Lebedev, A. Caneschi, and D. Gatteschi, Europhys. Lett. 44, 778 (1998).

[23] W. Wernsdorfer and R. Sessoli, Science 284, 133 (1999).

[24] M. Belesi, E. Micotti, M. Mariani, F. Borsa, A. Lascialfari, S. Carretta, P. Santini, G. Amoretti, E. J. L. McInnes, I. S. Tidmarsh, and J. R. Hawkett, Phys. Rev. Lett. 102, 177201 (2009).

[25] G. Aromi, and E. K. Brechin, Struct. Bonding 122, 1 (2006).

[26] M. N. Leuenberger, and D. Loss, Nature (London) 410, 789 (2001).

[27] F. Troiani, M. Affronte, S. Carretta, P. Santini, and G. Amoretti, Phys. Rev. Lett. 94, 190501 (2005).

[28] L. Bogani, and W. Wernsdorfer, Nat. Mater. 7, 179 (2008).

[29] F. Branzoli P. Carretta, M. Filibian, G. Zoppellaro, M. J. Graf, J. R. Galan-Mascaros, O. Fuhr, S. Brink, and M. Ruben, J. Am. Chem. Soc. 131, 4387 (2009).

[30] J. M. Clemente-Juan, E. Coronado, and A. Gaita-Ariño, Chem. Soc. Rev. 41, 7464 (2012); M. A. AlDamen J. M. Clemente-Juan, E. Coronado, C. Martì-Gastaldo, and A. Gaita-Arino, J. Am. Chem. Soc. 130, 8874 (2008); M. A. AlDamen, S. CardonaSerra, J. M. Clemente-Juan, E. Coronado, A. Gaita-Arino, C. Martì-Gastaldo, F. Luis, and O. Montero, Inorg. Chem. 48, 3467 (2009).

[31] M. Shiddiq, D. Komijani, Y. Duan, A. Gaita-Ariño, E. Coronado, and S. Hill, Nature 531, 348 (2016); M. D. Jenkins, Y. Duan, B. Diosdado, J. J. García-Ripoll, A. Gaita-Ariño, C. Giménez-Saiz, P. J. Alonso, E. Coronado, and F. Luis, Phys. Rev. B 95, 064423 (2017).

[32] F. Luis, M. J. Martínez-Pérez, O. Montero, E. Coronado, S. Cardona-Serra, C. Marti-Gastaldo, J. M. Clemente-Juan, J. Sesé, D. Drung, and T. Schurig, Phys. Rev. B 82, 060403(R) (2010).

[33] F.-S. Guo, B. M. Day, Y.-C. Chen, M.-L. Tong, A. Mansikkamki, and R. A. Layfield, Angew. Chem. Int. Ed. 56, 11445 (2017).

[34] C. A. P. Goodwin, F. Ortu, D. Reta, N. F. Chilton, and D. P. Mills, Nature 548, 439 (2017).

[35] N. Ishikawa, M. Sugita, T. Ishikawa, S. Koshihara, and Y. Kaizu, J. Phys. Chem. B 108, 11265 (2004). 
[36] N. Ishikawa, M. Sugita, and W. Wernsdorfer, J. Am. Chem. Soc. 127, 3650 (2005).

[37] N. Ishikawa, M. Sugita, and W. Wernsdorfer, Angew. Chem. Int. Ed. 44, 2931 (2005).

[38] M. Belesi, A. Lascialfari, D. Procissi, Z. H. Jang, and F. Borsa, Phys. Rev. B 72, 014440 (2005).

[39] P. Khuntia, M. Mariani, M. C. Mozzati, L. Sorace, F. Orsini, A. Lascialfari, F. Borsa, C. Maxim, and M. Andruh, Phys. Rev. B 80, 094413 (2009).

[40] D. Procissi, B. J. Suh, A. Lascialfari, F. Borsa, A. Caneschi, and A. Cornia, J. Appl. Phys. 91, 7173 (2002).

[41] J. H. Van Vleck, Phys. Rev. 74, 1168 (1948).

[42] F. Borsa, A. Lascialfari, and Y. Furukawa, in Novel NMR and EPR Techniques, edited by J. Dolinsek, M. Vilfan, and S. Zumer (Springer, New York, 2006).

[43] C. P. Slichter, Principles of Magnetic Resonance (SpringerVerlag, New York, 1996).

[44] H. Amiri, M. Mariani, A. Lascialfari, F. Borsa, G. A. Timco, F. Tuna, and R. E. P. Winpenny, Phys. Rev. B 81, 104408 (2010).

[45] T. Moriya, Prog. Theor. Phys. 16, 23 (1956).

[46] S. H. Baek, M. Luban, A. Lascialfari, E. Micotti, Y. Furukawa, F. Borsa, J. van Slageren, and A. Cornia, Phys. Rev. B 70, 134434 (2004).

[47] P. Santini, S. Carretta, E. Liviotti, G. Amoretti, P. Carretta, M. Filibian, A. Lascialfari, and E. Micotti, Phys. Rev. Lett. 94, 077203 (2005).

[48] H. Amiri, A. Lascialfari, Y. Furukawa, F. Borsa, G. A. Timco, and R. E. P. Winpenny, Phys. Rev. B 82, 144421 (2010).

[49] D. Procissi, A. Lascialfari, E. Micotti, M. Bertassi, P. Carretta, Y. Furukawa, and P. Kogerler, Phys. Rev. B 73, 184417 (2006);
J. Lago, E. Micotti, M. Corti, A. Lascialfari, A. Bianchi, S. Carretta, P. Santini, D. Procissi, S. H. Baek, P. Kogerler, C. Baines, and A. Amato, ibid. 76, 064432 (2007); S. J. Blundell, Contemp. Phys. 48, 275 (2007); T. Lancaster, J. S. Möller, S. J. Blundell, F. L. Pratt, P. J. Baker, T. Guidi, G. A. Timco, and R. E. P. Winpenny, J. Phys.: Condens. Matter 23, 242201 (2011); T. Lancaster, S. J. Blundell, F. L. Pratt, I. Franke, A. J. Steele, P. J. Baker, Z. Salman, C. Baines, I. Watanabe, S. Carretta, G. A. Timco, and R. E. P. Winpenny, Phys. Rev. B 81, 140409(R) (2010); A. Keren, O. Shafir, E. Shimshoni, V. Marvaud, A. Bachschmidt, and J. Long, Phys. Rev. Lett. 98, 257204 (2007).

[50] S. Sanna, P. Arosio, L. Bordonali, F. Adelnia, M. Mariani, E. Garlatti, C. Baines, A. Amato, K. P. V. Sabareesh, G. Timco, R. E. P. Winpenny, S. J. Blundell, and A. Lascialfari, Phys. Rev. B 96, 184403 (2017).

[51] A. Lascialfari, Z. H. Jang, F. Borsa, P. Carretta, and D. Gatteschi, Phys. Rev. Lett. 81, 3773 (1998).

[52] M. J. Graf, A. Lascialfari, F. Borsa, A. M. Tkachuk, and B. Barbara, Phys. Rev. B 73, 024403 (2006).

[53] R. Kubo and T. Toyabe, Magnetic Resonance and Relaxation (North Holland, Amsterdam, 1967), p. 810.

[54] J. J. Baldovì, J. M. Clemente-Juan, E. Coronado, Y. Duan, A. Gaita-Arino, and C. Gimenez-Sainz, Inorg. Chem. 53, 9976 (2014); or further details see also J. M. Clemente-Juan, E. Coronado, and A. Gaita-Arino, in Lanthanides and Actinides in Molecular Magnetism, edited by R. A. Layfield and M. Murugesu (Wiley-VCH, Weinheim, 2015).

[55] S. H. Baek, F. Borsa, Y. Furukawa, Y. Hatanaka, S. Kawakami, K. Kumagai, B. J. Suh, and A. Cornia, Phys. Rev. B 71, 214436 (2005). 